\title{
Effects of a Vestibular Stimulation Training Program on the Gait of Chronic Stroke Patients
}

\author{
Ki-Won Nam, PT, PhD • Jae-Chung Go, PT, $\mathrm{MS}^{1} \cdot$ Yong-Pil Yang, PT, $\mathrm{PhD}^{\dagger}$ \\ Dept. of Physical Therapy, Dongshin University \\ ${ }^{1}$ MediSPA Center of Dongshin University
}

Received: October 30, 2018 / Revised: November 5, 2018 / Accepted: January 10, 2019

(C) 2019 J Korean Soc Phys Med

\begin{abstract}
| Abstract |
PURPOSE: This study examined the effects of a vestibular stimulation training program on the walking ability of chronic stroke patients over a six month period.
\end{abstract}

METHODS: Forty stroke patients were enrolled in this study. The patients were divided randomly into a control group $(n=20)$ and experimental group $(n=20)$. A general exercise program was applied to Group I and vestibular stimulation training was applied to Group II (30 min, three times a week for six weeks). The changes in straight walking ability, curved walking ability, and functional walking ability were measured using a $10 \mathrm{~m}$ walking test figure-ofeight-walking test, and dynamic gait index, respectively. The measures before and after the program were compared using a paired t-test for a comparison of each group and an independent t-test for a comparison between groups.

RESULTS: The changes in each group were examined

†Corresponding Author : Yong-pil Yang raonpt@dsu.ac.kr, https://orcid.org/0000-0002-9398-623X

This is an Open Access article distributed under the terms of the Creative Commons Attribution Non-Commercial License (http://creativecommons.org/licenses/by-nc/3.0) which permits unrestricted non-commercial use, distribution, and reproduction in any medium, provided the original work is properly cited. according to the measurement period. The Experimental group showed significant functional improvement in all three tests after the vestibular stimulation training program, but the control group did not show significant improvement in any of the tests after the general exercise program. A comparison of the changes between groups revealed the experimental group to show significantly higher improvement than the control group in all tests.

CONCLUSION: The vestibular stimulation training program helps improve the gait function of stroke patients. Based on the results of this study, it is expected that various vestibular stimulation training programs will be developed and applied in a range of places.

Key Words: Exercise therapy, Gait, Stroke rehabilitation

\section{Introduction}

Stroke is a disorder, in which the blood supply to the brain is blocked or hemorrhage occurs in the brain tissue, resulting in an interrupted blood supply to the brain tissue [1]. Approximately $10 \%$ of stroke patients show complete recovery, but $15 \%$ die shortly after stroke, $65 \%$ require help with serious disabilities, and $10 \%$ are treated in a rehabilitation facility[2]. 
With the recent development of modern medicine and the improvements in living standards, the mortality rate has decreased gradually. On the other hand, there might be some disability depending on the area of injury of the brain, and long-term medical care is necessary to prevent secondary damage and improve the dysfunction [3].

Stroke manifests as reduced mobility caused by weakness in muscle strength, perception disorder, rigidity, and damage to the sensory organs and equilibrium[4,5]. This reduced mobility restricts the abilities to perform functional activities, such as gait, climbing stairs, standing and, changing direction, which are important for an independent daily life.

Of these restricted abilities, independent walking ability is one of the most important therapeutic goals of hemiplegic patients, which is considered as a basic condition to decide to return to their daily life after hospitalization[6]. Proprioceptive neuromuscular facilitation[7], weight shift training[8], task-oriented training[9], action observation training[10], and treadmill waking training[11] have been conducted to improve the patients' walking and balance ability.

When the vestibular system is affected, the visual and proprioceptive senses are impaired or central nervous and musculoskeletal system are impaired, which results in the incorrect passing of information. In addition, an integrating ability disorder can occur, which causes confusion in the spatial recognition and posture control commands in the central region, adversely affecting equilibrium and gait, dizziness, posture instability, etc., which makes therapeutic intervention and rehabilitation difficult $[12,13]$.

The vestibular system interacts with various regions, and influences eye movements and posture control. Therefore, a vestibular sensation can be stimulated and the problem can be improved through an interaction of the vestibular senses, visual, and proprioception[14].

In addition, functional inputs from the vestibular organ, proprioceptive sensory, and visual information systems are required to maintain effective balance[15]. Therefore, a number of studies have reported improvements in balance ability by applying a vestibular stimulation training program to adults with central nervous system impairment and children with cerebral palsy[16,17].

To control the posture required for walking, the vestibular system plays a very important role in recognizing and maintaining the balance of the body. Therefore, it is essential to study the effects of vestibular stimulation training on the balance and gait ability of stroke patients and assist in the rehabilitation of stroke patients.

This study examined the effects of vestibular stimulation training on the walking ability of stroke patients to develop a rehabilitation program to improve the diversity of exercise therapy intervention and the function of stroke patients.

\section{Methods}

\section{Participants}

This study was conducted on 40 adult hemiplegic patients, who were hospitalized in Y hospital in Gwang-Ju and underwent therapeutic exercise from August 1, 2016 to November 30, 2017. Table 1 lists the general characteristics of the subjects. The selection criteria of the subjects are as follows:

1) those diagnosed with a stroke by a rehabilitation specialist through computed tomography,

2) those more than six months after the stroke,

3) those with a Brunnstrom recovery stage greater than three,

4) those who could understand the purpose of this study and voluntarily agreed to participate, and

5) those who can walk independently using assistive devices.

In this study, the walking ability of stroke patients was measured before providing the vestigial training program and general exercise program and after six weeks of intervention using the measurement tools as follows. 
Table 1. General characteristics of the subjects

\begin{tabular}{cccc}
\hline & Experimental $(\mathrm{n}=20)$ & Control $(\mathrm{n}=20)$ & $\mathrm{p}$ \\
\hline Age (years) & $57.80 \pm 11.35$ & $59.80 \pm 13.27$ & .721 \\
Height $(\mathrm{cm})$ & $167.50 \pm 3.81$ & $165.30 \pm 7.07$ & .398 \\
Weight $(\mathrm{kg})$ & $63.60 \pm 6.20$ & $62.70 \pm 10.51$ & .818 \\
Onset (month) & $28.92 \pm 56$ & $26.58 \pm 8.6$ & .625 \\
Gender (M/F) & $12 / 8$ & $11 / 9$ & - \\
\hline
\end{tabular}

${ }^{\mathrm{a}}$ Mean $\pm \mathrm{SD}$, Experimental: Vestibular sensory stimulus training group

Control: General exercise group

\section{Outcome measures}

\section{1) $10 \mathrm{~m}$ Walking Test $(10 \mathrm{mWT})$}

A $10 \mathrm{~m}$ walking test $(10 \mathrm{mWT})$ was conducted to measure the straight walking ability. A total distance of $14 \mathrm{~m}$ was marked on the floor of the treatment room. For safety under the observation of the measurer, the subjects were directed to walk $14 \mathrm{~m}$ from the floor of the treatment room at their normal speed. With the exception of the initial and final two meters, the time taken to walk $10 \mathrm{~m}$ was measured in seconds. The $10 \mathrm{mWT}$ was taken as an average of three measurements without practice before the measurement.

\section{2) Figure-of-eight Walk Test (F8WT)}

The figure-of-eight walk test (F8WT) was conducted to measure the curve walking ability. The subjects stood between cones and selected the direction of travel. They were directed to walk at a normal speed and then stop when they returned to the starting position. The measurement factors of F8WT were composed of time, step count, accuracy, and naturalness. In this study, however, the time and step counts except for the measurement accuracy and naturalness were measured. The walking measurements were repeated three times in each session to obtain an average value.

\section{3) Dynamic Gait Index (DGI)}

The dynamic gait index (DGI) was used to measure the functional walking ability. The DGI consists of eight items: walking, walking with a change in pace, walking whilst horizontally and vertically turning the head, walking with rotating axes, crossing obstacles and returning, and climbing stairs. The DGI score ranged from zero to three on a four-point scale, with zero indicating severe impairment and three indicating normal. The highest number of point was 24 points. A lower score indicated greater impairment of functional mobility. All measurements were performed after the demonstrator demonstrated the process to the subject.

\section{Exercise programs}

\section{1) Vestibular stimulation training program}

The vestibular stimulation training program consisted of three activities for vestibular sensory stimulation and input for 30 minutes based on the training method presented by Ayres[18] and Hwang[19]. A total of 18 sessions of the vestibular stimulation training program were conducted, for 30 minutes per session three times a week. The vestibular stimulation training program was adjusted to gradually increase the intensity, time, and frequency according to the condition of the stroke patient. The types of the three activities and methods of intervention were as follows. 
(1) Swiss ball Activity

For the anterior, posterior, left, and right rectilinear stimulation activities, a therapeutic ball (Therapeutic ball, Thera-band, USA) was used. When sitting on the ball, the knees and hips were at 90 中. Sitting on the ball, the anterior, posterior, left and right rectilinear movements were performed for five minutes each.

(2) Pro Rocker Board Activity

A 20-inch Pro Rocker Board (Pro Rocker Board, Fitter First, USA) was used for the balancing activities in the right posture, and an auxiliary table was placed on both sides to prevent falls.

\section{(3) Trapeze Activity}

A trapeze was used for the anterior, posterior, left, and right activities in the sitting position, keeping the legs crossed and not dragging on the ground. The anterior, posterior, left, and right movements were conducted for five minutes each in the sitting position using the trapeze.

\section{2) General exercises}

The general exercise programs was applied to the control group, which consisted of movements that did not stimulate the vestibular sense. The treatment period consisted of 18 cycles of exercise, three times a week, once every 30 minutes. Each movement was controlled by changing the stage and method of exercise according to the stroke patient's level. The general exercise programs were as follows.

(1) Warm-up and Cool-down exercise

Stretching and ROM (range of motion) exercises were performed by therapist in the supine position as a warm-up and cool-down exercise for five minutes each.
(2) Bridge exercise

These exercises were performed while lying on a stable floor, maintaining a lumbar neutral position, flexing both knees $\left(90^{\circ}\right)$, placing soles on the floor, maintaining balance while lifting the pelvis and putting both arms comfortably on the floor. For 10 minutes, the subject was instructed to pull his or her navel upwards and backwards to activate the external oblique muscle as much as possible.

\section{(3) Crunch exercise}

The subject was laid down in the supine position with a pillow placed under the knee to reduce lumbar lordosis, which blocks compensatory action of the flexors of hip joint, by placing a towel under the scapula. The position of a retracted scapula was then made abducted and upward rotated, which causes both shoulders to be relaxed, to prevent compensatory action of the pectoralis major. The patient exercised for 10 minutes to contract the abdominal muscle whilst keeping their head up as if staring away.

\section{Statistical Analysis}

The SPSS ver 20.0 for Windows statistical analysis program was used to analyze the data of the study. The general characteristics of the subjects were analyzed by descriptive statistics, and a Shapiro-Wilks test was used to test the normality of the two groups. A paired t-test was used to compare the pre- and post-test statistics in each group, and an independent t-test was used to compare the statistics between groups. The statistical significance level was set to $a=.05$.

\section{Results}

\section{Changes of $10 \mathrm{mWT}$}

A comparison of the $10 \mathrm{mWT}$ before and after the experiment revealed a statistically significant decrease only in the experimental group after six weeks of intervention compared to that before the intervention $(\mathrm{p}<.05)$ (Table 2). 
Table 2. Change in the variables

\begin{tabular}{ccccc}
\hline & & Pre & Post & $\mathrm{p}$ \\
\hline $10 \mathrm{mWT}$ & Experimental & $19.90 \pm 5.23^{\mathrm{a}}$ & $16.18 \pm 6.37$ & $.032^{*}$ \\
(unit; sec) & Control & $20.21 \pm 6.78$ & $19.42 \pm 7.54$ & .086 \\
& $\mathrm{p}$ & .079 & $.026^{*}$ & $.001^{*}$ \\
\hline Step in F8WT & Experimental & $26.53 \pm 6.73$ & $18.59 \pm 5.86$ & .094 \\
(unit; step) & Control & $25.96 \pm 5.75$ & $24.67 \pm 7.43$ & $.005^{*}$ \\
\hline Time in F8WT & $\mathrm{p}$ & .265 & $16.57 \pm 6.46$ & $.000^{*}$ \\
(unit; sec) & Experimental & $24.74 \pm 5.97$ & $22.54 \pm 7.76$ & .163 \\
\hline CGI & Control & $23.76 \pm 6.56$ & $.000^{*}$ & $.032^{*}$ \\
\hline
\end{tabular}

${ }^{\mathrm{a}} \mathrm{Mean} \pm \mathrm{SD},{ }^{*} \mathrm{p}<.05$, Experimental : Vestibular sensory stimulation training group

Control : General exercise group

2. Changes in the F8WT

\section{1) Changes in the number of steps}

Only the experimental group showed a significant decrease in the number of steps in the F8WT after six weeks of intervention compared to that before the intervention $(\mathrm{p}<.05)$ (Table 2).

\section{2) Changes in time required}

Only the experimental group showed a significant decrease in the F8WT after six weeks of intervention compared to that before the intervention (Table 2).

\section{Change of DGl}

The experimental group showed a significant decrease in the DGI after six weeks of intervention compared to that before the intervention but not the control group $(\mathrm{p}<.05)$. (Table 2).

\section{Discussion}

The balance ability and posture control of stroke patients are important factors in their posture and walking[20], and disability occurs as a result of sensory- motor impairment. Sensory abnormalities can occur in both left and right hemiparesis, which causes an unstable posture and difficulty in the activities of daily living, such as balance and gait[21].

For stroke patients, the ultimate goal of rehabilitation is independent and functional walking. For stroke patients with reduced ability to walk, an increase in walking speed leads to improvements in everyday life[22]. This study examined the effects of a program that stimulates the vestibular system involved in balance ability on the walking ability when applied to chronic stroke patients.

In this study, the $10 \mathrm{mWT}$ was measured to determine the change in speed in a straight walk. The vestibular training group showed a significant difference between before and after the experiment, and there was a statistically significant difference between the control and vestibular 
stimulation training group. The walking ability is based on efficient posture control, which is necessary to control the vestibular sense, vision, and proprioceptive sensation [23]. The vestibular stimulation training program of this study appears to have influenced the postural control by appropriately stimulating the vestibular sense.

Twenty to fifty percent of all steps in human daily life consist of redirecting movements[24]. On the other hand, stroke patients have difficulty in redirecting movements, which is related to the motor system that provides information on the head movement and position[25].

In this study, the number of steps and time were measured using the F8WT, which was designed to measure the walking ability in a curve. As a result, the vestibular stimulation training group showed a significant decrease in the number of steps and the time taken compared to the control group. The decrease in the number of steps and time at the same distance can be explained as an increase in stride. Generally, the faster the gait velocity, the shorter the double support period of the gait cycle. In other words, the period of supporting with one leg is longer, highlighting the positive influence on the vestibular system.

Nakamura et al.[26] confirmed that pusher behavior was reduced by applying galvanic vestibular stimulation to stroke patients with pusher behavior. As a result, the improvement in weight distribution of the left and right legs positively affected walking. In addition, the DGI score for measuring the functional gait ability was increased significantly in the vestibular training group, which is consistent with Jeong and Choi[27], who reported improvement in the balance and walking ability, including the DGI after vestibular stimulation despite being somewhat different in the vestibular stimulation method. Therefore, vestibular stimulation training was found to be helpful for improving the walking ability of stroke patients. Further study of vestibular stimulation will be helpful for stroke rehabilitation. Future studies should examine a range of patient populations and develop various vestibular stimulation training programs for functional improvement.

\section{Conclusion}

The vestibular sensory stimulation program was effective in improving the curved walk (F8WT) and straight walk $(10 \mathrm{mWT})$ ability of stroke patients and improved the DGI as a functional gait test. Therefore, the vestibular sensory stimulation program used in this study can be used in rehabilitation programs of stroke patients at home and hospitals.

\section{References}

[1] Sims NR, Muyderman H. Mitochondria, oxidative metabolism and cell death in stroke. Biochim Biophys Acta. 2010;1802(1):80-91.

[2] Baek SH, Park EJ, Cho MS, et al. Analysis of the Burden actors Influencing Quality of Life of Stroke Patients Caregivers. KOEM. 2016;10(5):227-34.

[3] Mozaffarian D, Benjamin EJ, Go AS, et al. Heart disease and stroke statistics-2015 update: a report from the American Heart Association. Circulation. 2015;131(4): e29-e322.

[4] Kim JS, Kwon OH. The effect of arm swing on gait in post-stroke hemiparesis. J Korean Soc Phys Med. 2002;7(1): 95-101.

[5] Van Wyk A, Eksteen CA, Becker PJ, et al. A Cross-sectional Survey and Cross-sectional Clinical Trial to Determine the Prevalence and Management of Eye Movement Disorders and Vestibular Dysfunction in Post-Stroke Patients in the Sub-Acute Phase: Protocol. Front Neurol. 2016;20(7):1-20.

[6] Ng MF, Tong RK, Li LS. A pilot study of randomized clinical controlled trial of gait training in subacute stroke patients with partial body-weight support electromechanical gait trainer and functional electrical 
stimulation: Six-month follow-up. Stroke. 2008;39(1): 154-60.

[7] Sharma V, Kaur J. Effect of core strengthening with pelvic proprioceptive neuromuscular facilitation on trunk, balance, gait, and function in chronic stroke. J Exerc Rehabil. 2017;13(2):200-5.

[8] Ko YJ, Lee HS. The Effect of Weight-shift Training with Hula Hoop on Weight Shift Change and Gait in Stroke Patients: A Cross-Sectional Pilot Study. J Korean Soc Phys Med. 2017;12(1):9-14.

[9] Knox M, Stewart A, Richards CL. Six hours of task-oriented training optimizes walking competency post stroke: a randomized controlled trial in the public health-care system of South Africa. Clin Rehabil. 2018;32(8): 1057-68.

[10] Yang YP, Kim SJ. The Effect of Action Observation with Observation Type on Limits of Stability and Dynamic Gait Ability in Stroke Patients. J Korean Soc Phys Med. 2017;12(1):76-74.

[11] Park JE, Lee JH, Cha YJ. The Effect of Treadmill Gait Training in an Adjusted Position from Functional Training System on Chronic Stroke Patients' Walking and Balance Ability. J Korean Soc Phys Med. 2017;12(1):35-42.

[12] Geurts AC, Ribbers GM, Knoop JA, et al. Identification of static and dynamic postural instability following traumatic brain injury. Arch Phys Med Rehabil. 1996;77(7):639-44.

[13] Horak FB, Shupert CL, Dietz V, et al. Horstmann G. Vestibular and somatosensory contributions to responses to head and body displacements in stance. Exp Brain Res. 1994;100(1):93-106.

[14] Nashner LM, Black FO, Wall C. Adaptation to altered support and visual conditions during stance: patients with vestibular deficits. J Neurosci. 1982;2(5):536-44.

[15] Janssen LJ, Verhoeff LL, Horlings CG, et al. Directional effects of biofeedback on trunk sway during gait tasks in healthy young subjects. Gait Posture. 2009;29(4): 575-81.

[16] Badke MB, Miedaner JA, Shea TA et al. Effects of vestibular and balance rehabilitation on sensory organization and dizziness handicap. Ann Otol Rhinol Laryngol Suppl. 2005;114(1):48-54.

[17] Park MO. The .ects of vestibular stimulation training on balance for the children with cerebral palsy. Dankook University. Dissertation of Master's Degree. 2009.

[18] Ayres AJ. Sensory integration dysfunction: test score constellations. Part II of a final project report. Sensory integration international, 1986.

[19] Hwang SS. The Effects of vestibular stimulation on balance and fundamental psychological process of children with central nervous system dysfunction. Dankook University. Dissertation of Master's Degree. 1998.

[20] Tyson SF, Hanley M, Chillala J, et al. Balance Disability After Stroke. Phys Ther. 2006;86(1):30-8.

[21] Smania N, Montagnana B, Faccioli S, et al. Rehabilitation of somatic sensation and related deficit of motor control in patients with pure sensory stroke. Arch Phys Med Rehabil, 2003;84(11):1692-702.

[22] Lamontagne A, Fung J. Faster is better implication for speed-intensive gait training after stroke. Stroke. 2004;35(11):2543-48.

[23] Maurer C, Schweigrat G, Mergner T. Pronounced over estimation of support surface tilt during stance. Exp Brain Res. 2006;168(12):41-50.

[24] Segal AD, Orendurff MS, Czerniecki JM, et al. Local dynamic stability in turning and straight-line gait. $\mathrm{J}$ biomech. 2008;41(7):1486-93.

[25] Carr JH, Shepherd RB. Neurological rehabilitation: optimizing motor performance(2nd ed). New York. Churchill Livingstone. 2010.

[26] Nakamura J, Kita Y, Yuda T, et al. Effects of galvanic vestibular stimulation combined with physical therapy on pusher behavior in stroke patients: a case series. NeuroRehabilitation. 2014;35(1):31-7.

[27] Jeong HY, Choi JD. The effects of vestibular sensory stimulation training on balance and gait in the patients with stroke. J Kor Phy Ther. 2014;26(5);365-71. 
